\title{
Exchange-correlation potentials in density-functional and spin-density-functional theory
}

\author{
Tai Kai Ng \\ Department of Physics, Massachusetts Institute of Technology, Massachusetts 02139
}

(Received 1 September 1988; revised manuscript received 1 December 1988)

\begin{abstract}
Exact expressions for the exchange-correlation potential $W_{\mathrm{xc}}(\mathbf{r})$ in density- and spin-densityfunctional theories are derived under a very general situation where $W_{\mathrm{xc}}(\mathbf{r})$ is expressed in terms of two- and three-particle correlation functions. Within the random-phase approximation it is shown that there exist nonlocal contributions to $W_{x c}(\mathbf{r})$ which cannot be reproduced in any "local-density" type of approximations (LDA). In the nonmagnetic case, "nonlocal" contributions are found to be present only in $V_{\mathrm{xc}}(\mathbf{q}=0)$, which leads to the famous "band-gap" problem. In the magnetic case it is found that nonlocality is present in all components of $W_{\mathrm{xc}}(\mathbf{r})$ in general, indicating that the LDA is in general an inadequate approximation for exchange-correlation potentials in magnetic systems. An alternative approximation for $W_{\mathrm{xc}}(\mathbf{r})$ is proposed which also includes nonlocality in an approximate way.
\end{abstract}

\section{INTRODUCTION}

The density-functional theory introduced by Hohenberg, Kohn, and Sham ${ }^{1,2}$ has proven to describe many ground-state properties of molecules and solids correctly. In this theory it is shown ${ }^{2}$ that the ground-state properties of inhomogeneous electron systems can be obtained by solving a single-particle Schrödinger equation for electrons moving in an effective potential $V_{\text {eff }}(\mathbf{r})$. The effective potential consists of a sum of the external potential $V^{\text {ext }}(\mathbf{r})$, the Hartree potential $\phi_{H}(\mathbf{r})$, and the exchange-correlation potential $V_{\mathrm{xc}}(\mathbf{r})$, which is defined as the functional derivative of the exchange-correlation energy $E_{\mathrm{xc}}[n]$ with respect to the local density $n(\mathbf{r})$. In practice, the exchange-correlation potential $V_{\mathrm{xc}}(\mathbf{r})$ is usually evaluated in the local-density approximation (LDA) where $E_{\mathrm{xc}}[n]$ is given in each small neighborhood of $\mathbf{r}$ by the exchange-correlation energy of the homogeneous electron gas at the same local density. This approximation was found to be successful in a lot of cases except perhaps in the calculation of semiconductor and insulator band gaps $^{3-5}$ and in some magnetic systems ${ }^{6,7}$ [local spin-density approximation (LSDA)]. The purpose of the present paper is to perform a general study of the exchange-correlation potentials in the density- and spindensity-functional theory. In particular we would like to have some general idea under what situation is the localdensity approximation (LDA or LSDA) going to be inadequate, and whether we can find simple enough corrections to LDA which can remedy the problem. Our basic idea is as follows: Based on an exact expression for the exchange-correlation energy $E_{\mathrm{xc}}$, it has been argued by several authors ${ }^{8-11}$ that LDA can provide a reasonable approximation for $E_{\mathrm{xc}}$. However, it does not necessarily imply that LDA would also provide a reasonable approximation for the derivative of $E_{\mathrm{xc}}$, with respect to the density change. For example, in the band-gap problem, the variation $\delta E_{\mathrm{xc}} / \delta n(0)$ across the band gap is discontinuous. ${ }^{4,5}$ However, this behavior is absent in
LDA which would always produce smooth changes in the derivative $\delta E_{\mathrm{xc}} / \delta n(\mathbf{r})$. Therefore, we shall try instead to derive an exact expression for the exchangecorrelation potential $V_{\mathrm{xc}}(\mathbf{r})$ itself and try to see whether we can find out from studying this exact expression some simple criteria for when the LDA is going to be inadequate. We shall restrict ourselves to the case of a perfect crystal with magnetic field pointing in the $\widehat{\mathbf{z}}$ direction in the present paper.

The organization of our paper is as follows. In Sec. II we derive a formally exact expression for the exchangecorrelation potentials in spin-density-functional theory under the above conditions [which include the nonmagnetic situation (density-functional theory) as a special case] where the exchange-correlation potentials are expressed in terms of the two-particle and three-particle correlation functions under the coupling constant integral. ${ }^{8-11}$ In analyzing these expressions, we find two kind of terms. The first kind of terms involve momentum transfer $\mathbf{q}=n \mathbf{Q}$ where $\mathbf{Q}$ is a reciprocal-lattice vector and $n$ is a nonzero integer whereas the second kind of terms involve momentum transfer $\mathbf{q} \rightarrow \mathbf{0}$. We argue that LDA can be reasonable only on the first kind of terms which involve electronic processes within a distance $r \lesssim 1 /$ $Q \sim a_{0}$ ( $a_{0}$ is equal to the lattice constant) whereas the second kind of terms are nonlocal in nature and have to be treated more carefully.

Our ideas are put on a more quantitative basis in Sec. III when the random-phase approximation (RPA) is introduced to calculate the various correlation functions. The nature of the nonlocal terms are pointed out and as an example we specialize ourselves to the nonmagnetic case and show that while nonlocality is in general unimportant in metals, the discontinuity in the $q=0$ component of the exchange-correlation potential in insulators as a function of electron density as one crosses the band gap $^{3-5}$ is indeed a natural consequence of the existence of a nonlocal type of term in the exchange-correlation potential.

In Sec. IV we discuss the general case of finite magneti- 
zation (within RPA) and point out that because of the existence of nonlocal terms, LDA is in general inadequate for constructing the exchange-correlation potential in magnetic systems. An alternative approximation is discussed where LDA is applied primarily only on the "local" terms in the expression for the exchange-correlation potential. The resulting expression for the exchangecorrelation potential consists of two terms: the LDA term and a correction term which depends on the selfenergies $\Sigma_{\sigma}\left(k_{F \sigma}, \mu\right)$ of the electronic states on the Fermi surface.

Finally, some discussions and comments will be given in Sec. V, where we shall discuss the validity of our approach under more general situations.

\section{EXACT EXPRESSION FOR THE EXCHANGE-CORRELATION POTENTIALS IN PERIODIC SYSTEMS}

In the following discussion we shall consider a perfect crystal in zero temperature. We shall also restrict ourselves to the case when the external magnetic field is pointing at the $\hat{\mathbf{z}}$ direction only. Extension of our theory to more general situations suffers from some technical difficulties which we shall discuss at the end of the paper.

For a periodic system it is more convenient to work in momentum space. The external potentials can be written as $[$ notice that $\mathbf{H}(\mathbf{r})=\hat{\mathbf{z}} H(\mathbf{r})]$

$$
\begin{aligned}
V_{\sigma}^{\mathrm{ext}}(\mathbf{r}) & =V(\mathbf{r})+\sigma H(\mathbf{r}) \\
& =\sum_{\mathbf{q} \in\{Q\}} V(\mathbf{q}) e^{i \mathbf{q} \cdot \mathbf{r}}+\sigma \sum_{\mathbf{q} \in\{0, Q\}} H(\mathbf{q}) e^{i \mathbf{q} \cdot \mathbf{r}},
\end{aligned}
$$

where $\sigma= \pm 1$ is the spin index. $\{Q\}$ and $\{0, Q\}$ represent the set of all reciprocal-lattice vectors with the zero vector excluded and included, respectively. Notice that a constant scalar potential has no physical effect on the system (which we have set to be zero for simplicity) whereas a constant magnetic field affects the relative distribution of the $\uparrow$ and $\downarrow$ spin electrons and thus has a definite physical meaning.

Correspondingly, we can introduce the particle density $n(\mathbf{r})$ and magnetization density $m(\mathbf{r})$ where

$$
n(\mathbf{r})=n_{\uparrow}(\mathbf{r})+n_{\downarrow}(\mathbf{r})=\sum_{\mathbf{q} \in\{0, Q\}} n(\mathbf{q}) e^{i \mathbf{q} \cdot \mathbf{r}}
$$

and

$$
m(\mathbf{r})=n_{\uparrow}(\mathbf{r})-n_{\downarrow}(\mathbf{r})=\sum_{\mathbf{q} \in\{0, Q\}} m(\mathbf{q}) e^{i \mathbf{q} \cdot \mathbf{r}} .
$$

The independent parameters which characterize a given system are the external potentials $\left.V\left(\mathbf{q}^{\prime}\right)\right|_{\mathbf{q}^{\prime} \in\{Q\}}$ and $\left.H\left(\mathbf{q}^{\prime}\right)\right|_{\mathbf{q}^{\prime} \in\{0, Q\}}$ and the mean particle density $n(0)$. In particular, we have

$$
\left.n(\mathbf{q})\right|_{\mathbf{q} \in\{Q\}}=n\left(\mathbf{q},\left[H\left(\mathbf{q}^{\prime \prime}\right)\right],\left[V\left(\mathbf{q}^{\prime}\right)\right], n(0)\right)
$$

and

$$
\left.m(\mathbf{q})\right|_{\mathbf{q} \in\{0, Q\}}=m\left(\mathbf{q},\left[H\left(\mathbf{q}^{\prime \prime}\right)\right],\left[V\left(\mathbf{q}^{\prime}\right)\right], n(0)\right) .
$$

Notice that $n(0)$ is an independent parameter whereas $m(\mathbf{q}=0)$ depends both on the external fields and $n(0)$.
Using Eq. (3) we can write

$$
\begin{aligned}
d n(\mathbf{q})_{\mathbf{q} \in\{Q\}}= & \left.\frac{\partial n(\mathbf{q})}{\partial n(0)}\right|_{H, V} d n(0) \\
& +\left.\sum_{\mathbf{q}^{\prime} \in\{Q\}} \frac{\partial n(\mathbf{q})}{\partial V\left(\mathbf{q}^{\prime}\right)}\right|_{H, n(0)} d V\left(\mathbf{q}^{\prime}\right) \\
& +\left.\sum_{\mathbf{q}^{\prime \prime} \in\{0, Q\}} \frac{\partial n(\mathbf{q})}{\partial H\left(\mathbf{q}^{\prime \prime}\right)}\right|_{V, n(0)} d H\left(\mathbf{q}^{\prime \prime}\right),
\end{aligned}
$$

and

$$
\begin{aligned}
\left.d m(\mathbf{q})\right|_{\mathbf{q} \in\{0, Q\}}= & \left.\frac{\partial m(\mathbf{q})}{\partial n(0)}\right|_{H, V} d n(0) \\
& +\left.\sum_{\mathbf{q}^{\prime} \in\{Q\}} \frac{\partial m(\mathbf{q})}{\partial V\left(\mathbf{q}^{\prime}\right)}\right|_{H, n(0)} d V\left(\mathbf{q}^{\prime}\right) \\
& +\left.\sum_{\mathbf{q}^{\prime \prime} \in\{0, Q\}} \frac{\partial m(\mathbf{q})}{\partial H\left(\mathbf{q}^{\prime \prime}\right)}\right|_{V, n(0)} d H\left(\mathbf{q}^{\prime \prime}\right),
\end{aligned}
$$

where from linear-response theory, we have

$$
\begin{aligned}
& \left.\frac{\partial \xi(\mathbf{q})}{\partial V\left(\mathbf{q}^{\prime}\right)}\right|_{H, n(0)}=\chi_{\xi n}\left(\mathbf{q}, \mathbf{q}^{\prime}\right), \\
& \left.\frac{\partial \xi(\mathbf{q})}{\partial H\left(\mathbf{q}^{\prime}\right)}\right|_{V, n(0)}=\chi_{\xi m}\left(\mathbf{q}, \mathbf{q}^{\prime}\right) .
\end{aligned}
$$

$\xi=n, m$ are the particle- and magnetization-density variables, and

$$
\chi_{\xi \xi^{\prime}}\left(\mathbf{q},-\mathbf{q}^{\prime}\right)=\frac{1}{2 \pi} \int_{-\infty}^{\infty} d t\left\langle T \xi(\mathbf{q}, t) \xi^{\prime}\left(\mathbf{q}^{\prime}, 0\right)\right\rangle
$$

$\left(\xi, \xi^{\prime}=n, m\right)$ is the static response function associated with the density variables $\xi$ and $\xi^{\prime}$.

By applying a generalized theorem of Mearn and Kohn ${ }^{12}$ to the present situation (see Appendix A), the inverse functions of $\chi_{\xi \xi^{\prime}}\left(\mathbf{q}, \mathbf{q}^{\prime}\right)$ exist and are defined by

$$
\sum_{\substack{\xi^{\prime}=n, m \\ \mathbf{q}^{\prime \prime} \in\left\{\xi^{\prime}\right\}}} \chi_{\xi \xi^{\prime}}^{-1}\left(\mathbf{q}, \mathbf{q}^{\prime \prime}\right) \chi_{\xi^{\prime} \xi^{\prime \prime}}\left(\mathbf{q}^{\prime \prime}, \mathbf{q}^{\prime}\right)=\delta\left(\mathbf{q}-\mathbf{q}^{\prime}\right) \delta_{\xi \xi^{\prime \prime}},
$$

where $\left\{\xi^{\prime}=n\right\}=\{Q\}$ and $\left\{\xi^{\prime}=m\right\}=\{0, Q\}$. The vectors $\mathbf{q}$ and $\mathbf{q}^{\prime}$ are defined over the sets $\{\xi\}$ and $\left\{\xi^{\prime \prime}\right\}$, respectively. Notice that the HK theorem is defined for a system with fixed number of particles, which results in the absence of the $q=0$ particle-density component in the definition of the inverse $\chi^{-1}$.

Combining Eqs. (4) and (7), we obtain

$$
\begin{aligned}
\left.d W(\mathbf{q})\right|_{\mathbf{q} \in\left\{\xi_{W}\right\}}= & \sum_{\substack{\xi^{\prime}=n, m \\
\mathbf{q}^{\prime} \in\left\{\xi^{\prime}\right\}}} \chi_{\xi_{W} \xi^{\prime}}^{-1}\left(\mathbf{q}, \mathbf{q}^{\prime}\right) \\
& \times\left[d \xi^{\prime}\left(\mathbf{q}^{\prime}\right)-\left.\frac{\partial \xi\left(\mathbf{q}^{\prime}\right)}{\partial n(0)}\right|_{H, V} d n(0)\right],
\end{aligned}
$$

where $W=V, H, \quad \xi_{V}=n$, and $\xi_{H}=m$. Notice that $\left.\delta n(\mathbf{q})\right|_{\mathbf{q} \in\{0, Q\}}$ and $\left.\delta m(\mathbf{q})\right|_{\mathbf{q} \in\{0, Q\}}$ now form a complete set of variables describing small fluctuations of the physi- 
cal system near the ground state.

Like all other physical quantities the exchangecorrelation energy $E_{\mathrm{xc}}$ is also a functional of $V(q), H(q)$, and $n(0)$. Therefore,

$$
d E_{\mathrm{xc}}=\left.\sum_{\substack{W=H, V \\ \mathbf{q}^{\prime} \in\left\{\xi_{W}\right\}}} \frac{\partial E_{\mathrm{xc}}}{\partial W\left(\mathbf{q}^{\prime}\right)}\right|_{n(0)} d W\left(\mathbf{q}^{\prime}\right)+\left.\frac{\partial E_{\mathrm{xc}}}{\partial n(0)}\right|_{W} d n(0)
$$

Combining Eqs. (8) and (9), we find

$$
\begin{aligned}
\left.\frac{1}{\Omega} \frac{\partial E_{\mathrm{xc}}}{\partial n(\mathbf{q})}\right|_{\mathbf{q} \in\{Q\}} & \\
= & V_{\mathrm{xc}}(\mathbf{q})=\left.\frac{1}{\Omega} \sum_{\substack{W=H, V \\
\mathbf{q}^{\prime} \in\left\{\xi_{W}\right\}}} \frac{\partial E_{\mathrm{xc}}}{\partial W\left(\mathbf{q}^{\prime}\right)}\right|_{n(0)} \chi_{\xi_{W^{n}}^{-1}\left(\mathbf{q}^{\prime}, \mathbf{q}\right),},
\end{aligned}
$$

$$
\begin{aligned}
& \left.\frac{1}{\Omega} \frac{\partial E_{\mathrm{xc}}}{\partial m(\mathbf{q})}\right|_{\mathbf{q} \in\{0, Q\}} \\
& \quad=H_{\mathrm{xc}}(\mathbf{q})=\left.\frac{1}{\Omega} \sum_{\substack{W=H, V \\
\mathbf{q}^{\prime} \in\left\{\xi_{W}\right\}}} \frac{\partial E_{\mathrm{xc}}}{\partial W\left(\mathbf{q}^{\prime}\right)}\right|_{n(0)} \chi_{\xi_{W} m^{-1}\left(\mathbf{q}^{\prime}, \mathbf{q}\right),}
\end{aligned}
$$

and

$$
\begin{aligned}
\frac{1}{\Omega} \frac{\partial E_{\mathrm{xc}}}{\partial n(0)}= & V_{\mathrm{xc}}(0) \\
= & \left.\frac{\partial E_{\mathrm{xc}}}{\partial n(0)}\right|_{H, V}-\left.\sum_{\mathbf{q}^{\prime} \in\{Q\}} V_{\mathrm{xc}}\left(\mathbf{q}^{\prime}\right) \frac{\partial n\left(\mathbf{q}^{\prime}\right)}{\partial n(0)}\right|_{H, V} \\
& -\left.\sum_{\mathbf{q}^{\prime} \in\{0, Q\}} H_{\mathrm{xc}}\left(\mathbf{q}^{\prime}\right) \frac{\partial m\left(\mathbf{q}^{\prime}\right)}{\partial n(0)}\right|_{H, V}, \quad(10 \mathrm{c})
\end{aligned}
$$

where $\Omega$ is the volume of the system.
Notice that the variation $\partial E_{\mathrm{xc}} / \partial n(0)$ on the left-hand side of Eq. (10c) is performed with all other $\mathbf{q}$ components of the particle density and magnetization density fixed, while the corresponding term on the right-hand side is performed at fixed external potentials. Notice that in adding (or removing) an electron (with the external potential fixed), the particle density does not change uniformly in general (also the magnetization density changes). The effects coming from these changes have to be subtracted off in defining $V_{\mathrm{xc}}(0)$, which is the physical origin of the second and third terms in right-hand side of Eq. (10c).

To evaluate the partial derivatives $\partial E_{\mathrm{xc}} / \partial W(\mathbf{q})$ and $\partial E_{\mathrm{xc}} / \partial n(0)$ in Eq. (10), we make use of an exact expression for $E_{\mathrm{xc}}$ where $E_{\mathrm{xc}}$ is expressed in terms of the paircorrelation function:

$$
E_{\mathrm{xc}}=\frac{1}{2} \int d \mathbf{r}_{1} \int d \mathbf{r}_{2} \frac{1}{\left|\mathbf{r}_{1}-\mathbf{r}_{2}\right|} \int_{0}^{1} d \lambda\left\langle n\left(\mathbf{r}_{1}\right) n\left(\mathbf{r}_{2}\right)\right\rangle_{\lambda}^{c}
$$

where for simplicity we have set $e^{2}=1$. The function $\left\langle n\left(\mathbf{r}_{1}\right) n\left(\mathbf{r}_{2}\right)\right\rangle_{\lambda}^{c}$ is defined as follows: ${ }^{8-11}$ let $n(\mathbf{r})$ and $m(\mathbf{r})$ be the true ground-state particle- and magnetization-density distributions of the physical system of electrons under the external potential $V(\mathbf{r})$ and $H(\mathbf{r})$ and with Coulomb interaction between electrons $\left|\mathbf{r}_{1}-\mathbf{r}_{2}\right|^{-1}$. Now consider the interaction term multiplied by a factor $\lambda(<1)$, and $V(\mathbf{r})$ and $H(\mathbf{r})$ changed to $V_{\lambda}(\mathbf{r})$ and $H_{\lambda}(\mathbf{r})$ such that the density distributions $n(\mathbf{r})$ and $m(\mathbf{r})$ remain unchanged. We have

$$
\begin{aligned}
\left\langle n\left(\mathbf{r}_{1}\right) n\left(\mathbf{r}_{2}\right)\right\rangle_{\lambda}^{c}= & \left\langle n\left(\mathbf{r}_{1}\right) n\left(\mathbf{r}_{2}\right)\right\rangle_{\lambda} \\
& -\delta\left(\mathbf{r}_{1}-\mathbf{r}_{2}\right)\left\langle n\left(\mathbf{r}_{1}\right)\right\rangle_{\lambda} \\
& -\left\langle n\left(\mathbf{r}_{1}\right)\right\rangle_{\lambda}\left\langle n\left(\mathbf{r}_{2}\right)\right\rangle_{\lambda},
\end{aligned}
$$

where $\langle A\rangle_{\lambda}$ denotes the expectation value of $A$ in the ground state corresponding to $V_{\lambda}(\mathbf{r})$ and $H_{\lambda}(\mathbf{r})$ and with interaction $\lambda\left|\mathbf{r}_{1}-\mathbf{r}_{2}\right|^{-1}$. Notice that fixing $n(\mathbf{r})$ and $m(\mathbf{r})$ implies that the Kohn-Sham potentials $V^{\mathrm{KS}}(\mathbf{r})$ and $H^{\mathrm{KS}}(\mathrm{r})$ are also independent of $\lambda$ by the HK theorem.

Fourier transforming Eq. (11), we obtain

$$
E_{\mathrm{xc}}=\frac{1}{2} \int d \mathbf{r}_{1} \int d \mathbf{r}_{2} \frac{1}{\left|\mathbf{r}_{1}-\mathbf{r}_{2}\right|} \frac{1}{(2 \pi)^{3}} \int d \mathbf{q} \sum_{\mathbf{q}^{\prime} \in\{0, Q\}} e^{i \mathbf{q} \cdot \mathbf{r}_{1}} e^{i\left(\mathbf{q}^{\prime}-\mathbf{q}\right) \cdot \mathbf{r}_{2}} \int_{0}^{1} d \lambda\left\langle n(\mathbf{q}) n\left(\mathbf{q}^{\prime}-\mathbf{q}\right)\right\rangle_{\lambda}^{c},
$$

where we have made use of the periodicity condition in obtaining Eq. (13).

With Eqs. (10) and (13), what remains to be evaluated are the derivatives $\partial\left\langle n(\mathbf{q}) n\left(\mathbf{q}^{\prime}-\mathbf{q}\right)\right\rangle_{\lambda}^{c} / \partial W\left(\mathbf{q}^{\prime \prime}\right)$ and $\partial\left\langle n(\mathbf{q}) n\left(\mathbf{q}^{\prime}-\mathbf{q}\right)\right\rangle_{\lambda}^{c} / \partial n(0)$ (assuming that the order of integration and differentiation can be interchanged for different variables), which can again be obtained via linear-response theory,

$$
\begin{aligned}
& \frac{\partial\left\langle n(\mathbf{q}) n\left(\mathbf{q}^{\prime}-\mathbf{q}\right)\right\rangle_{\lambda}^{c}}{\partial W\left(\mathbf{q}^{\prime \prime}\right)} \\
& =\chi_{n n ; \xi_{W}}\left(\mathbf{q}, \mathbf{q}^{\prime}-\mathbf{q} ; \mathbf{q}^{\prime \prime}\right)-\chi_{n \xi_{W}}\left(\mathbf{q}^{\prime}, \mathbf{q}^{\prime \prime}\right) \\
& =\chi_{n n ; \xi_{W}}\left(\mathbf{q}, \mathbf{q}^{\prime}-\mathbf{q} ; \mathbf{q}^{\prime \prime}\right),
\end{aligned}
$$

where 
$\chi_{n n ; \xi_{W}}\left(\mathbf{q}_{1}, \mathbf{q}_{2} ; \mathbf{q}_{3}\right)$

$$
=\frac{1}{2 \pi} \int_{-\infty}^{\infty} d t\left\langle\operatorname{Tn}\left(\mathbf{q}_{1}, t\right) n\left(\mathbf{q}_{2}, t\right) \xi_{W}\left(\mathbf{q}_{3}, 0\right)\right\rangle,
$$

$\chi_{n \xi_{W}}$ being the static response function defined in Eq. (5). To evaluate the derivative $\partial / \partial n(0)$, we observe that

$$
\left.\frac{\partial}{\partial n(0)}\right|_{W}=\left.\left.\frac{\partial \mu}{\partial n(0)}\right|_{W} \frac{\partial}{\partial \mu}\right|_{W},
$$

where $\mu$ is the chemical potential. Notice also that the variation of $\mu$ is equivalent to the negative of the variation of a static external scalar potential $\phi e^{i \mathbf{q} \cdot \mathbf{r}}$ in the limit $\mathbf{q} \rightarrow 0 .{ }^{13}$ Therefore,

$$
\left.\frac{\partial}{\partial n(0)}\right|_{W}=-\left.\left.\frac{\partial \mu}{\partial n(0)}\right|_{W} \frac{\partial}{\partial V(\mathbf{q})}\right|_{\mathbf{q} \rightarrow 0} .
$$

Combining Eqs. (10), (13), (14), and (16), we obtain

$$
\begin{aligned}
\left.W_{\mathrm{xc}}(\mathbf{q})\right|_{W=H, V, \mathbf{q} \in\left\{\xi_{W}\right\}}=\frac{1}{2 \Omega} \int d \mathbf{r}_{1} \int & d \mathbf{r}_{2} \frac{1}{\left|\mathbf{r}_{1}-\mathbf{r}_{2}\right|} \frac{1}{(2 \pi)^{3}} \\
& \times \int d \mathbf{q}^{\prime} \sum_{\mathbf{q}^{\prime \prime} \in\{0, Q\}} e^{i \mathbf{q}^{\prime} \cdot \mathbf{r}_{1}} e^{i\left(\mathbf{q}^{\prime \prime}-\mathbf{q}^{\prime}\right) \cdot \mathbf{r}_{2}} \\
& \times \int_{0}^{1} d \lambda \sum_{\substack{W^{\prime}=H, V \\
\mathbf{q}^{\prime \prime \prime} \in\left\{\xi_{W^{\prime}}\right\}}} \chi_{\lambda n n ; \xi_{W^{\prime}}}^{c}\left(\mathbf{q}^{\prime}, \mathbf{q}^{\prime \prime}-\mathbf{q}^{\prime} ; \mathbf{q}^{\prime \prime \prime}\right) \chi_{\lambda \xi_{W^{\prime}} \xi_{W}}^{-1}\left(\mathbf{q}^{\prime \prime \prime}, \mathbf{q}\right),
\end{aligned}
$$

and

$$
\begin{aligned}
& V_{\mathrm{xc}}(0)=\frac{1}{2 \Omega} \int d \mathbf{r}_{1} \int d \mathbf{r}_{2} \frac{1}{\left|\mathbf{r}_{1}-\mathbf{r}_{2}\right|} \frac{1}{(2 \pi)^{3}} \\
& \times \int d_{\mathbf{q}^{\prime}} \sum_{\mathbf{q}^{\prime \prime} \in\{0, Q\}} e^{i \mathbf{q}^{\prime} \cdot \mathbf{r}_{1}} e^{i\left(\mathbf{q}^{\prime \prime}-\mathbf{q}^{\prime}\right) \cdot \mathrm{r}_{2}} \\
& \times \int_{0}^{1} \frac{d \lambda}{\chi_{\lambda}(0)} \mid \chi_{\lambda n n ; n}^{c}\left(\mathbf{q}^{\prime}, \mathbf{q}^{\prime \prime}-\mathbf{q}^{\prime} ; \mathbf{q} \rightarrow 0\right) \\
& \left.-\sum_{\substack{W ; W^{\prime}=H, V \\
\mathbf{q}^{\prime \prime \prime} \in\left\{\xi_{W}\right\} \\
\mathbf{q}^{\prime \prime \prime \prime} \in\left\{\xi_{W^{\prime}}\right\}}}\left[\chi_{\lambda n n ; \xi_{W}}^{c}\left(\mathbf{q}^{\prime}, \mathbf{q}^{\prime \prime}-\mathbf{q}^{\prime} ; \mathbf{q}^{\prime \prime \prime}\right) \chi_{\lambda \xi_{W} \xi_{W^{\prime}}}^{-1}\left(\mathbf{q}^{\prime \prime \prime}, \mathbf{q}^{\prime \prime \prime \prime}\right) \chi_{\lambda \xi_{W^{\prime}}}\left(\mathbf{q}^{\prime \prime \prime \prime}, q \rightarrow 0\right)\right]\right],
\end{aligned}
$$

where

$$
\begin{gathered}
\left.\frac{\partial n(0)}{\partial \mu}\right|_{W}=-\left.\frac{\partial n(0)}{\partial V(\mathbf{q})}\right|_{\mathbf{q} \rightarrow 0}=-\chi_{n n}(0, \mathbf{q} \rightarrow 0) \\
=-\chi(0)
\end{gathered}
$$

Equations (17) and (18) constitute the basic result obtained in this section which we shall analyze in the following. First of all, we notice that instead of formal derivatives, the exchange-correlation potentials are expressed in terms of correlation functions now, which are more ready for systematic methods of treatment, although the introduction of $\lambda$-dependent external fields make straightforward perturbation treatment difficult. ${ }^{14}$ We notice also that because of the condition of periodicity, the momentum transfers which appear in the correlation functions are always restricted to the set $\{0, Q\}$, except the integral $\int d \mathbf{q}^{\prime}$ which runs over all values of $\mathbf{q}^{\prime}$. Notice that normally electronic processes which contribute to an excitation with momentum transfer $\mathbf{q}$ are mainly coming from a region in $\mathbf{r}$ space with $|\mathbf{r}| \lesssim 1 /|\mathbf{q}|$. Thus with $\mathbf{q} \sim \mathbf{Q},|\mathbf{r}| \lesssim a_{0}$ ( $a_{0}$ is approximately equal to the lattice constant) and most of the physical processes which determine $W_{\mathrm{xc}}(\mathrm{q})$ are "local" in character. The only exceptions are the processes involving momentum transfer $\mathbf{q} \rightarrow 0$ and the ones under the $\int d \mathbf{q}^{\prime}$ integral. However, the $\int d \mathbf{q}^{\prime}$ integral represents the sum over all processes coming from the exchange-correlation hole around an electron. ${ }^{8-11}$ The size of the exchange-correlation hole is typically of order $r \sim 1 / k_{F} \sim a_{0}$ which is also shortranged in character. Thus the only kind of processes which are truly "nonlocal" in character are the ones associated with momentum transfer $q \rightarrow 0$. These processes involve electrons traveling throughout the whole crystal and thus LDA or its generalization which depend on "local" properties ${ }^{15,16}$ are not expected to be reasonable approximations for these terms (unless the electron density of the crystal itself is close to uniform). It is interesting to point out also that in the nonmagnetic case $\left[H(\mathbf{q}), H_{\mathrm{xc}}(\mathbf{q}) \equiv 0\right]$, the nonlocal $\mathbf{q} \rightarrow 0$ terms are present in the $q=0$ component of the exchange-correlation potential only [see Eqs. (17) and (18)]. Thus one expects LDA to be a good approximation for nonmagnetic systems under most situations where $V_{\mathrm{xc}}(\mathrm{q}=0)$ is not of interest $\left[V_{\mathrm{xc}}(0)\right.$ only renormalize the chemical potential and have no direct effect on the density distribution $n(\mathbf{r})$ 
or the Kohn-Sham energy spectrum], which seems to be consistent with results obtained from band-structure calculations using LDA in density-functional theory. ${ }^{3,11,17}$

In the following sections, Eqs. (17) and (18) will be analyzed in the Random-phase approximation (RPA) in which our ideas are put on a more quantitative basis. We shall see that some interesting results are indeed obtained.

\section{RPA AND DISCONTINUITY IN $V_{\mathrm{xc}}(0)$ ACROSS THE BAND GAP}

The RPA can be defined as follows: First we express all two-particle and three-particle correlation functions in Eqs. (17) and (18) in terms of "proper" correlation functions, which are defined as the sum of all Feynman diagrams (with the correct external constraints characterizing the correlation function) which cannot be separated into two parts by removing any one Coulomb line. ${ }^{18}$ The full two-particle and three-particle correlation functions can then be expressed as the sums of "proper" correlation functions joined by single Coulomb lines. This is shown schematically in Figs. 1(a) and 1(b).

In RPA, the "proper" correlation function $\chi_{\lambda}^{(p)}$ is replaced by $\chi_{0 \lambda}$, where $\chi_{0 \lambda}$ is the corresponding correlation function for a system of noninteracting electrons moving in "appropriately" chosen external potentials $\bar{V}_{\lambda}(\mathbf{r})$ and $\bar{H}_{\lambda}(\mathbf{r})$. Notice that there is some ambiguity in the definition of $\bar{V}_{\lambda}$ and $\bar{H}_{\lambda},{ }^{14}$ which comes from different ways of doing perturbation expansion. Here we shall take $\bar{V}_{\lambda}(\mathbf{r})=V^{\mathrm{KS}}(\mathbf{r})$ and $\bar{H}_{\lambda}(\mathbf{r})=H^{\mathrm{KS}}(\mathbf{r})$ (i.e., the KohnSham potentials) which is independent if $\lambda$ [see discussion after Eq. (12)]. This choice is more consistent with our way of defining $E_{\mathrm{xc}}$ and is also physically more reasonable. With this approximation, it is now possible to examine the exchange-correlation potentials more quantitatively. As an example, we shall consider first of all the case of nonmagnetic systems in this section. The general case of magnetic systems will be examined in the next section.

For nonmagnetic systems, $H_{\mathrm{xc}}(\mathrm{q}) \equiv 0$, and the exchange-correlation potential is given by Eqs. (17) and (18) with $W(\mathbf{q})=V(\mathbf{q})$ only. In RPA, it can be shown after some algebra (see Appendix B) that

$$
\begin{aligned}
& \sum_{\mathbf{q}^{\prime \prime \prime} \in\{Q\}} \chi_{\lambda n n ; n}^{c}\left(\mathbf{q}^{\prime}, \mathbf{q}^{\prime \prime}-\mathbf{q}^{\prime} ; \mathbf{q}^{\prime \prime \prime}\right) \chi_{\lambda n n}^{-1}\left(\mathbf{q}^{\prime \prime \prime}, \mathbf{q}\right) \\
& =\frac{1}{\beta} \sum_{i \Omega_{n} \mathbf{Q}^{\prime}, \mathbf{Q}^{\prime \prime} \in\{0, Q\}} \sum_{\mathbf{Q}^{\prime \prime \prime} \in\{Q\}} \frac{1}{\epsilon_{\lambda}\left(\mathbf{q}^{\prime}, \mathbf{Q}^{\prime}+\mathbf{q}^{\prime}, i \Omega_{n}\right)} \frac{1}{\epsilon_{\lambda}\left(\mathbf{q}^{\prime \prime}-\mathbf{q}^{\prime}, \mathbf{Q}^{\prime \prime}-\mathbf{q}^{\prime},-i \Omega_{n}\right)} \\
& \quad \times \chi_{0 n n}\left(\mathbf{Q}^{\prime}+\mathbf{q}^{\prime}, i \Omega_{n}, \mathbf{Q}^{\prime \prime}-\mathbf{q}^{\prime},-i \Omega_{n} ; \mathbf{Q}^{\prime \prime \prime}, 0\right) \chi_{0 n n}^{-1}\left(\mathbf{Q}^{\prime \prime \prime}, \mathbf{q}\right)-\delta\left(\mathbf{q}-\mathbf{q}^{\prime \prime}\right),
\end{aligned}
$$

and

$$
\begin{aligned}
& \chi_{\lambda n n ; n}^{c}\left(\mathbf{q}^{\prime}, \mathbf{q}^{\prime \prime}-\mathbf{q}^{\prime} ; q \rightarrow 0\right)-\sum_{\mathbf{q}^{\prime \prime \prime}, \mathbf{q}^{\prime \prime \prime \prime} \in\{Q\}} \chi_{\lambda n n ; n}^{c}\left(\mathbf{q}^{\prime}, \mathbf{q}^{\prime \prime}-\mathbf{q}^{\prime}, \mathbf{q}^{\prime \prime \prime}\right) \chi_{\lambda n n}^{-1}\left(\mathbf{q}^{\prime \prime \prime}, \mathbf{q}^{\prime \prime \prime \prime}\right) \chi_{\lambda n n}\left(\mathbf{q}^{\prime \prime \prime \prime}, \mathbf{q} \rightarrow 0\right) \\
& =\frac{1}{\beta} \sum_{i \Omega_{n} \mathbf{Q}^{\prime}, \mathbf{Q}^{\prime \prime} \in\{0, Q\}} \frac{1}{\epsilon_{\lambda}\left(\mathbf{q}^{\prime}, \mathbf{Q}^{\prime}+\mathbf{q}^{\prime}, i \Omega_{n}\right)} \frac{1}{\epsilon_{\lambda}\left(\mathbf{q}^{\prime \prime}-\mathbf{q}^{\prime}, \mathbf{Q}^{\prime \prime}-\mathbf{q}^{\prime},-i \Omega_{n}\right)} \\
& \times\left(\chi_{0 n n}\left(\mathbf{Q}^{\prime}+\mathbf{q}^{\prime}, i \Omega_{n}, \mathbf{Q}^{\prime \prime}-\mathbf{q}^{\prime},-i \Omega_{n} ; \mathbf{q} \rightarrow 0,0\right)\right. \\
& \left.-\sum_{\mathbf{q}^{\prime \prime \prime}, \mathbf{q}^{\prime \prime \prime \prime} \in\{Q\}} \chi_{0 n n ; n}\left(\mathbf{Q}^{\prime}+\mathbf{q}^{\prime}, i \Omega_{n}, \mathbf{Q}^{\prime \prime}-\mathbf{q}^{\prime},-i \Omega_{n} ; \mathbf{q}^{\prime \prime \prime}, 0\right) \chi_{0 n n}^{-1}\left(\mathbf{q}^{\prime \prime \prime}, \mathbf{q}^{\prime \prime \prime \prime}\right) \chi_{0 n n}\left(\mathbf{q}^{\prime \prime \prime \prime}, \mathbf{q} \rightarrow 0\right)\right] \text {, }
\end{aligned}
$$

where

$$
\frac{1}{\epsilon\left(\mathbf{q}, \mathbf{q}^{\prime}, i \Omega\right)}=\delta\left(\mathbf{q}-\mathbf{q}^{\prime}\right)+\phi(\mathbf{q}) \chi_{n n}\left(\mathbf{q}, \mathbf{q}^{\prime}, i \Omega\right)
$$

is the inverse of the dielectric function $\epsilon\left(\mathbf{q}, \mathbf{q}^{\prime}, i \Omega\right)$. $\phi\left(q^{\prime}\right)=4 \pi / q^{\prime 2}$ is the Coulomb potential. $\chi_{n n}\left(\mathbf{q}, \mathbf{q}^{\prime}, i \Omega\right)$ is the density-density response function at finite frequency $i \Omega$. $\epsilon$ and $\chi$ are evaluated in the RPA (see Appendix B). $\chi_{0 n n ; n}$ and $\chi_{0 n n}$ are the three-particle and two-particle density correlation-functions, respectively, for the system of noninteracting electrons moving in the KS potential.

As was argued in the last section, we expect that in the nonmagnetic case, LDA is a reasonable approximation for $V_{\mathrm{xc}}(\mathrm{q})$ except for the $\mathrm{q}=0$ component. Thus we shall first consider $V_{\mathrm{xc}}(0)$. This term is unimportant in metal- lic compounds since it only renormalizes the chemical potential. An example is the system of a homogeneous electron gas in a uniform positive background charge where the chemical potential is a continuous function of density and the properties of the system are unaffected by its absolute magnitude. This term is of physical significance in the insulator band-gap problem ${ }^{3-5}$ where the band gap is defined as $E_{g}=\mu^{+}-\mu^{-}$, where $\mu^{+}$and $\mu^{-}$are the chemical potentials at zero temperature, when the system has one electron in the conduction band $\left(\mu^{+}\right)$ and one hole in the valence band $\left(\mu^{-}\right)$, respectively. ${ }^{4,5}$

We shall first consider the "nonlocal" $(q \rightarrow 0)$ components in $V_{\mathrm{xc}}(0)$. From Eqs. (18) and (20), it is easy to show that only two "nonlocal" terms are present in $V_{\mathrm{xc}}(0)$, 
(a)
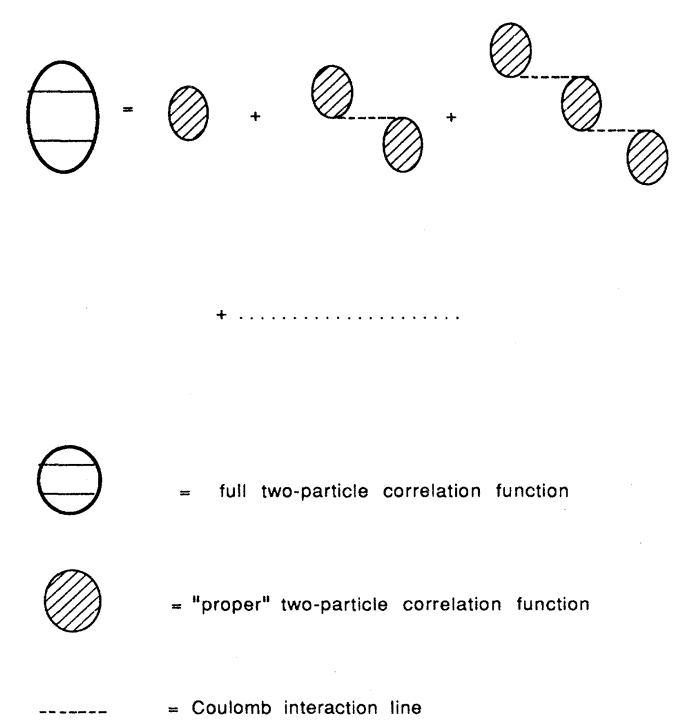

(b)
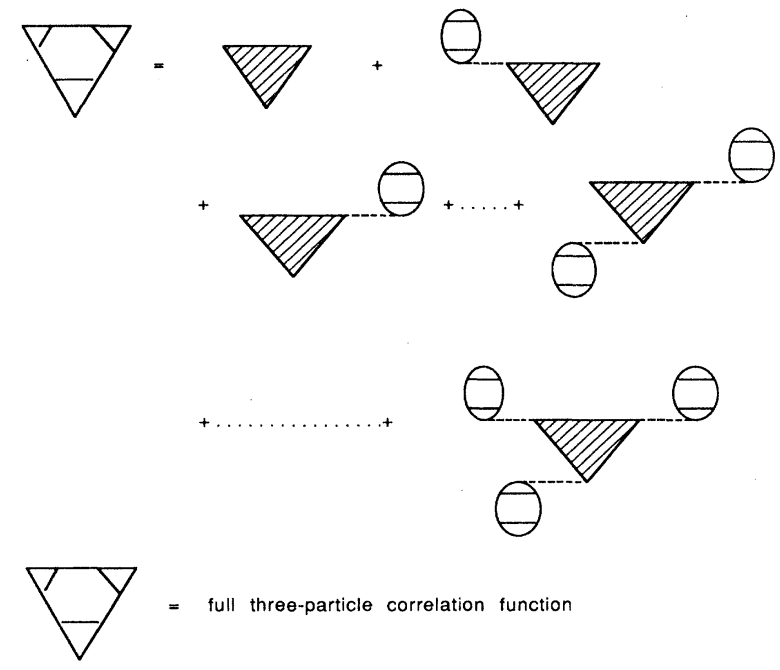

= full three-particle correlation function

$=$ "proper" three-particle correlation function

FIG. 1. Schematic representation of how Feynman diagrams can be considered as pieces of "proper" diagrams connected by single Coulomb lines for (a) a two-particle correlation function and (b) a three-particle correlation function.

$$
\chi_{0 n n ; n}\left(\mathbf{Q}^{\prime}+\mathbf{q}^{\prime}, i \Omega_{n}, \mathbf{Q}^{\prime \prime}-\mathbf{q}^{\prime},-i \Omega_{n}, \mathbf{q} \rightarrow 0,0\right)
$$

and

$$
\chi_{0 n n}\left(\mathbf{q}^{\prime \prime \prime \prime}, \mathbf{q} \rightarrow 0\right)
$$

To examine these two terms, it is simplest to use the identities [see Eq. (16)]

$$
\begin{aligned}
\chi_{0 n n ; n}\left(\mathbf{q}^{\prime}, i \omega, \mathbf{q}^{\prime \prime},\right. & -i \omega ; \mathbf{q} \rightarrow 0,0) \\
= & -\left.\frac{\partial}{\partial \mu} \chi_{0 n n}\left(\mathbf{q}^{\prime}, i \omega, \mathbf{q}^{\prime \prime},-i \omega\right)\right|_{V},
\end{aligned}
$$

and

$$
\chi_{0 n n}\left(\mathbf{q}^{\prime}, \mathbf{q} \rightarrow 0,0\right)=-\left.\frac{\partial}{\partial \mu} n\left(\mathbf{q}^{\prime}\right)\right|_{V} .
$$

For a system of noninteracting electrons, these derivatives are easy to perform with the results

$$
\begin{aligned}
& \chi_{0 n n ; n}\left(\mathbf{q}^{\prime}, i \omega, \mathbf{q}^{\prime \prime},-i \omega, \mathbf{q} \rightarrow 0,0\right) \\
&=\frac{-1}{V^{2}} \sum_{\mathbf{k}, \mathbf{k}^{\prime}} {\left[\frac{\delta\left(\mu-\varepsilon_{k}\right)-\delta\left(\mu-\varepsilon_{k^{\prime}}\right)}{\varepsilon_{k}-\varepsilon_{k^{\prime}}-i \omega}\right) } \\
& \times \times \rho_{k^{\prime} k}\left(\mathbf{q}^{\prime}\right) \rho_{k k^{\prime}}\left(\mathbf{q}^{\prime \prime}\right),
\end{aligned}
$$

and

$$
\chi_{0 n n}\left(\mathbf{q}^{\prime}, \mathbf{q} \rightarrow 0\right)=\frac{-1}{V} \sum_{k} \delta\left(\mu-\varepsilon_{k}\right) \rho_{k k}\left(\mathbf{q}^{\prime}\right),
$$

where

$$
\rho_{k k^{\prime}}(\mathbf{q})=2 \int d \mathbf{r} e^{i \mathbf{q} \cdot \mathbf{r}} \psi_{k}(\mathbf{r}) \psi_{k^{\prime}}^{*}(\mathbf{r}),
$$

$\psi_{k}$ being the eigenstates for the system of noninteracting electrons moving in the KS potential with energy $\varepsilon_{k}$. The factor 2 is coming from spin degeneracy.

The particular nature of these "nonlocal" components is now clear. They involve only contributions from electronic states on the Fermi surface. It is also clear why these terms will give rise to a discontinuity in $V_{\mathrm{xc}}(0)$ as one crosses the band gap. In the case when there is one hole in the valence band, the $\delta\left(\mu-\varepsilon_{k}\right)$ term in Eqs. (23) and (24) picks up the wave function $\psi_{k}^{v}(\mathbf{r})$ which is at the top of the valence band, while in the case when there is one electron at the conduction band, it picks up the wave function $\psi_{k}^{c}(\mathbf{r})$ which is at the bottom of the conduction band. The difference between $\psi_{k}^{v}(\mathbf{r})$ and $\psi_{k}^{c}(\mathbf{r})$ is of order 1 which results in a discontinuous change in the nonlocal components of $V_{\mathrm{xc}}(0)$ as one crosses the band gap. (For a full account of the application of our present theory to band-gap problems, see Ref. 19. We found that our approach is equivalent to the Sham-Schlüter ${ }^{4,20}$ theory in this case, see also Ref. 21 and Sec. IV.) It is also clear that any local-density type of approximation is bad for these "nonlocal" terms since it is impossible to represent an individual wave function which is extended in space in terms of properties local in space in general.

In contrast, one can show using Eqs. (19) and (20) that the "local" components which enter into $V_{\mathrm{xc}}(\mathbf{q})\left(\mathbf{q}^{\prime} \neq 0\right.$ processes) always involve electronic processes covering the whole Fermi sphere. To see why this is so, let us consider the term $\chi_{0 n n}\left(\mathbf{q}, \mathbf{q}^{\prime}\right)$ with $\mathbf{q}, \mathbf{q}^{\prime} \in\{Q\}$ which gives the density response of the system to a weak external potential $V\left(\mathbf{q}^{\prime}\right)$. In general, an external potential with wave vector $\mathbf{q}^{\prime}$ will disturb all states with momentum $k_{F}-q^{\prime} \leq|\mathbf{k}| \leq k_{F}+q^{\prime}$. With $\quad \mathbf{q}^{\prime} \sim Q \sim k_{F}, \quad$ essentially states in the whole Fermi sphere are disturbed. One consequence of this is that the discontinuity in $V_{\mathrm{xc}}(\mathbf{q})$ across the band gap can appear in the "nonlocal" components, i.e., $V_{\mathrm{xc}}(0)$, only. This is because the "local" terms always involve disturbance of the whole Fermi surface, and the effect of discontinuity in one electronic state will be of order $O(1 / N)(N$ is the total number of elec- 
trons), which is negligible as $N \rightarrow \infty$.

Notice that in LDA, roughly speaking, one is replacing the true Fermi sphere locally by Fermi spheres of homogeneous electron gas, in a way such that the correct distribution of electrons is obtained. This approximation is meaningful for electronic processes which involve the whole Fermi sphere; the error comes mainly from a quantitative difference in the shape of the Fermi sphere. However, the approximation is unjustifiable in describing individual electronic states, which can have a qualitatively very different character from plane-wave states when external potentials are switched on (for example, electronic states close to a band gap). Contributions to the exchange-correlation potentials from individual states are present in general in the form of nonlocal terms, as can be seen from our analysis, and they should be treated more carefully to obtain a reliable approximation for the exchange-correlation potentials. This is the subject of the next section.

\section{EXCHANGE-CORRELATION POTENTIAL IN GENERAL (MAGNETIC) SITUATIONS}

In this section we shall examine the general exchangecorrelation potentials [Eqs. (17) and (18)] within RPA. We shall first consider the exchange-correlation potentials $\left.V_{\mathrm{xc}}(\mathbf{q})\right|_{\mathrm{q} \in\{Q\}}$ and $\left.H_{\mathrm{xc}}(\mathbf{q})\right|_{\mathrm{q} \in\{0, Q\}} \cdot V_{\mathrm{xc}}(0)$ will be studied afterward. Using Eq. (17) and following procedures as described in the last section (see also Appendix B), we obtain after some algebra

$$
\begin{aligned}
\left.W_{\mathrm{xc}}(\mathbf{q})\right|_{W=H, V, \mathbf{q} \in\left\{\xi_{W}\right\}}= & \frac{1}{2 \Omega} \int d \mathbf{r}_{1} \int d \mathbf{r}_{2} \frac{1}{\left|\mathbf{r}_{1}-\mathbf{r}_{2}\right|} \frac{1}{(2 \pi)^{3}} \int d \mathbf{q}^{\prime} \sum_{\mathbf{q}^{\prime \prime} \in\{0, Q\}} e^{i\left(\mathbf{q}^{\prime \prime}-\mathbf{q}^{\prime}\right) \cdot \mathbf{r}_{2}} e^{i \mathbf{q}^{\prime} \cdot \mathbf{r}_{1}} \\
& \times \int_{0}^{1} d \lambda\left[\frac{1}{\beta} \sum_{i \Omega_{n} \mathbf{Q}^{\prime}, \mathbf{Q}^{\prime \prime} \in\{0, Q\}} \sum_{\sum_{\lambda}\left(\mathbf{q}^{\prime}, \mathbf{Q}^{\prime}+\mathbf{q}^{\prime}, i \Omega_{n}\right)} \frac{1}{\epsilon_{\lambda}\left(\mathbf{q}^{\prime \prime}-\mathbf{q}^{\prime}, \mathbf{Q}^{\prime \prime}-\mathbf{q}^{\prime},-i \Omega_{n}\right)}\right] \\
& \times \sum_{\substack{W^{\prime}=H, V \\
\mathbf{q}^{\prime \prime \prime} \in\left\{\xi_{W^{\prime}}\right\}}} \chi_{0 n n ; \xi_{W^{\prime}}}\left(\mathbf{Q}^{\prime}+\mathbf{q}^{\prime}, i \Omega_{n}, \mathbf{Q}^{\prime \prime}-\mathbf{q}^{\prime},-i \Omega_{n} ; \mathbf{q}^{\prime \prime \prime}, 0\right) \\
& \left.\times \widetilde{\chi}_{\lambda \xi_{W^{\prime}} \xi_{W}}\left(\mathbf{q}^{\prime \prime \prime}, \mathbf{q}\right)-\delta_{\xi_{W^{n}}} \delta\left(\mathbf{q}-\mathbf{q}^{\prime \prime}\right)\right],
\end{aligned}
$$

where

$\tilde{\chi}_{\lambda \xi_{W^{\prime}} \xi_{W}}^{-1}\left(\mathbf{q}^{\prime}, \mathbf{q}\right)=\delta_{\xi_{W^{n}}} \delta_{\xi_{W^{\prime}}} \delta\left(\mathbf{q}^{\prime}-\mathbf{q}\right) \phi(\mathbf{q})+\chi_{\lambda \xi_{W^{\prime}} \xi_{W}}^{-1}\left(\mathbf{q}^{\prime}, \mathbf{q}\right)$

$\phi(\mathbf{q})=4 \pi / q^{2}$ is the Coulomb potential. $\epsilon$ 's are the dielectric functions and $\chi^{-1}$,s are the inverses of the response functions as defined in Eqs. (19)-(21). All response functions and their inverses are evaluated in RPA in our present approximation (see Appendix B). As in the previous section we shall first consider the "nonlocal" $(\mathbf{q} \rightarrow \mathbf{0})$ contributions. On examining Eq. (26) we find that there are two sources of nonlocality. First of all, the term

$$
\chi_{0 n n ; m}\left(\mathbf{Q}^{\prime}+\mathbf{q}^{\prime}, i \Omega_{n}, \mathbf{Q}^{\prime \prime}-\mathbf{q}^{\prime},-i \Omega_{n}, \mathbf{q}^{\prime \prime \prime} \rightarrow 0,0\right)
$$

is expected to be nonlocal $\left(\mathbf{q}^{\prime \prime \prime} \rightarrow 0\right)$. Secondly, $\mathbf{q} \rightarrow 0$ terms also appear in the inverse response functions in Eq. (26). In fact, on closer examination one finds that nonlocality actually appears in $\chi^{-1}$ in all momentum transfers, since although nonlocal contributions appear in $\chi$ in momentum transfer $\mathbf{q} \rightarrow 0$ only, they get mixed up in all $\mathbf{q}$ components in $\chi^{-1}$ as a result of the inverse operation. Notice that the $q \rightarrow 0$ processes which contribute to $W_{\mathrm{xc}}(\mathrm{q})$ are all magnetic in origin. This is a direct consequence of the fact that the HK theorem is defined for fixed particle density $n(0)$ but not fixed $m(0)$ (see Sec. II).

To evaluate the nonlocal terms, it is most easy to follow a procedure similar to the nonmagnetic case [Eqs. (22) $-(25)]$. Here we shall consider first the term $\chi_{0 n n ; m}$,

$$
\begin{aligned}
\chi_{0 n n ; m}\left(\mathbf{q}, i \omega, \mathbf{q}^{\prime},-i \omega ; \mathbf{q}^{\prime \prime \prime} \rightarrow 0,0\right) \\
=\frac{\partial}{\partial H} \chi_{0 n n}\left(\mathbf{q}, \mathbf{q}^{\prime}, i \omega\right) \\
=\sum_{\sigma} \frac{\partial \mu_{\sigma}}{\partial H} \frac{\partial}{\partial \mu_{\sigma}} \chi_{0 \sigma}\left(\mathbf{q}, \mathbf{q}^{\prime}, i \omega\right),
\end{aligned}
$$

where $\chi_{0 n n}$ is the $\omega$-dependent density-density response function for the system of noninteracting electron gas moving in the $\mathrm{KS}$ potentials and $\chi_{0 \sigma}$ is the corresponding response function for electrons with spin component $\sigma$. It is tempting to identify $\partial \mu_{\sigma} / \partial H=-\mu_{B} \sigma$ for a system of noninteracting electrons. However, this is incorrect because the derivative in Eq. (28) is defined for fixed $n(0)$. An additional shift in chemical potential is also found which compensates the change in $n(0)$ under the change of the magnetic field, i.e., we have

$$
\frac{\partial n(0)}{\partial H}=\sum_{\sigma} \frac{\partial n_{\sigma}}{\partial \mu_{\sigma}} \frac{\partial \mu_{\sigma}}{\partial H}=0 \text {. }
$$

Writing $\partial \mu_{\sigma} / \partial H=\mu_{B}(\delta-\sigma)$, where $\delta$ is spin independent, we find from Eq. (29)

$$
\delta=\frac{\sum_{\sigma} \sigma \frac{\partial n_{\sigma}}{\partial \mu_{\sigma}}}{\sum_{\sigma} \frac{\partial n_{\sigma}}{\partial \mu_{\sigma}}}=\frac{N_{\uparrow}(0)-N_{\downarrow}(0)}{N_{\uparrow}(0)+N_{\downarrow}(0)},
$$

where $N_{\sigma}(0)$ is the density of state for the electrons with 
spin component $\sigma$ on the Fermi surface. Combining this with Eq. (28), we obtain

$$
\begin{aligned}
\chi_{0 n n ; m}\left(\mathbf{q}, i \omega, \mathbf{q}^{\prime},-i \omega ; \mathbf{q}^{\prime \prime \prime} \rightarrow 0,0\right) \\
=\mu_{B} \sum_{\sigma= \pm 1}(\delta-\sigma) \frac{1}{\Omega^{2}} \\
\times \sum_{k, k^{\prime}}\left[\frac{\delta\left(\mu-\varepsilon_{k \sigma}\right)-\delta\left(\mu-\varepsilon_{k^{\prime} \sigma}\right)}{\varepsilon_{k \sigma}-\varepsilon_{k^{\prime} \sigma}-i \omega}\right] \\
\quad \times \rho_{k^{\prime} k}^{\sigma}(\mathbf{q}) \rho_{k k^{\prime}}^{\sigma}\left(\mathbf{q}^{\prime}\right),
\end{aligned}
$$

where

$$
\rho_{k k^{\prime}}^{\sigma}(\mathbf{q})=\int d \mathbf{r} e^{i \mathbf{q} \cdot \mathbf{r}} \psi_{k \sigma}(\mathbf{r}) \psi_{k^{\prime} \sigma}^{*}(\mathbf{r}),
$$

$\psi_{k \sigma}(\mathbf{r})$ being the eigenstates of the system of noninteracting electrons with spin $\sigma$ and energy $\varepsilon_{k \sigma}$, moving under the KS potentials.

As in the nonmagnetic case, the nonlocal terms depend explicitly on the electronic states on the Fermi surface [a parallel analysis of the nonlocal terms appearing in $\chi$ (or $\chi^{-1}$ ) arrives at the same conclusion]. However, unlike the nonmagnetic case, the nonlocal terms appear in all momentum components in the exchange-correlation potentials now. Thus, we expect LDA to be in general inadequate for constructing exchange-correlation potentials in magnetic systems, which seems to be in agreement with general conclusions drawn from band-structure calculations using SLDA. ${ }^{6,7}$ In the following, we shall try to construct an alternative approximation for the exchange-correlation potential which is not too difficult to use but with nonlocality treated more carefully.

An obvious possibility would be to make the LDA only on those terms in the expression for $W_{\mathrm{xc}}(\mathbf{q})$ which are local in character and evaluate the other terms "exactly." However, even this approximation is extremely complicated because of the complicated way nonlocal terms appear in $\chi^{-1}$. To keep the approximation simple, we shall simply assume that $\chi^{-1}$ is well approximated by LDA. A justification of this approximation is that each element in $\chi^{-1}$ involves a complicated mixture of a large number of elements in $\chi$, of which the number of "local" elements is much larger than the number of "nonlocal" elements (only one row and one column in the matrix $\chi$ is nonlocal since nonlocality associates with $\mathbf{q} \rightarrow 0$ processes only). Thus one expects that the inverse $\chi^{-1}$ is dominated by "local" elements. With this approximation, nonlocality in the exchange-correlation potentials appears only through the term $\chi_{0 n n ; m}\left(\mathbf{q}, i \omega, \mathbf{q}^{\prime},-i \omega, \mathbf{q}^{\prime \prime \prime} \rightarrow 0,0\right)$. Going back to Eq. (26), we find that the "nonlocal" component in $W_{\mathrm{xc}}(\mathrm{q})$ is given (in this approximation) by

$$
\begin{aligned}
\left.W_{\mathrm{xc}}^{\mathrm{NL}}(\mathbf{q})\right|_{\mathbf{q} \in\left\{\xi_{W}\right\}}= & \frac{1}{2 \Omega} \int d \mathbf{r}_{1} \int d \mathbf{r}_{2} \frac{1}{\left|\mathbf{r}_{1}-\mathbf{r}_{2}\right|} \frac{1}{(2 \pi)^{3}} \int d \mathbf{q}^{\prime} \sum_{\mathbf{q}^{\prime \prime} \in\{0, Q\}} e^{i\left(\mathbf{q}^{\prime \prime}-\mathbf{q}^{\prime}\right) \cdot \mathbf{r}_{2}} e^{i \mathbf{q}^{\prime} \cdot \mathbf{r}_{1}} \\
& \times \int_{0}^{1} d \lambda \frac{1}{\beta} \sum_{i \Omega_{n} \mathbf{Q}^{\prime}, \mathbf{Q}^{\prime \prime} \in\{0, Q\}} \sum_{\left.\frac{1}{\epsilon_{\lambda}\left(\mathbf{q}^{\prime}, \mathbf{Q}^{\prime}+\mathbf{q}^{\prime}, i \Omega_{n}\right)} \frac{\tilde{\chi}_{\lambda m, \xi_{W}}^{-1}(0, \mathbf{q})}{\epsilon_{\lambda}\left(\mathbf{q}^{\prime \prime}-\mathbf{q}^{\prime}, \mathbf{Q}^{\prime \prime}-\mathbf{q}^{\prime},-i \Omega_{n}\right)}\right)_{\mathbf{L D A}}} \\
& \times \chi_{0 n n ; m}\left(\mathbf{Q}^{\prime}+\mathbf{q}^{\prime}, i \Omega_{n}, \mathbf{Q}^{\prime \prime}-\mathbf{q}^{\prime},-i \mathbf{\Omega}_{n}, \mathbf{q}^{\prime \prime \prime} \rightarrow 0,0\right)
\end{aligned}
$$

where the terms in the large parentheses are "local" and are evaluated approximately in LDA. The term $\chi_{0 n n ; m}$ is evaluated "exactly" with Kohn-Sham wave functions on the Fermi surface. The resulting exchange-correlation potential can be written as

$\left.W_{\mathrm{xc}}(\mathbf{q})\right|_{\mathbf{q} \in\left\{\xi_{W}\right\}}=W_{\mathrm{xc}}^{\mathrm{LDA}}(\mathbf{q})+\left[W_{\mathrm{xc}}^{\mathrm{NL}}(\mathbf{q})-W_{\mathrm{xc}}^{\mathrm{NL}(\mathrm{LDA})}(\mathbf{q})\right]$,

where $W_{\mathrm{xc}}^{\mathrm{NL}(\mathrm{LDA})}(\mathbf{q})$ is given by Eq. (32) with $\chi_{0 n n ; m}$ also evaluated in LDA. Equation (32) can be further simplified. We shall not go into the mathematical details here, but instead write down the final result. The deriva- tion is given in Appendix C. We find that $W_{\mathrm{xc}}^{\mathrm{NL}}(\mathbf{q})$ can be written as

$$
W_{\mathrm{xc}}^{\mathrm{NL}}(\mathbf{q})=-\mu_{B} \sum_{\sigma= \pm 1} \sigma \widetilde{N}\left\langle\Sigma_{\sigma}^{\mathrm{RPA}}(k, \mu) \bar{\chi}_{m \xi_{W}}^{-1(\mathrm{LDA})}(0, \mathbf{q})\right.
$$

where

$$
\widetilde{N}=\frac{2 N_{\uparrow}(0) N_{\downarrow}(0)}{N^{\uparrow}(0)+N_{\downarrow}(0)}
$$

and

$$
\Sigma_{\sigma}^{\mathrm{RPA}}(k, \omega)=\left.\frac{-1}{\Omega} \int d \mathbf{r} \int d \mathbf{r}^{\prime} \psi_{k \sigma}\left(\mathbf{r}^{\prime}\right) \psi_{k \sigma}^{*}\left(\mathbf{r}^{\prime}\right) \frac{1}{\beta} \sum_{i \Omega_{n}} V_{\mathrm{eff}}^{\mathrm{RPA}}\left(\mathbf{r}-\mathbf{r}^{\prime}, i \Omega_{n}\right)\right|_{n=n(\mathbf{r}), m=m(\mathbf{r})} g_{\sigma}^{0}\left(\mathbf{r}^{\prime}, \mathbf{r}, \omega-i \Omega_{n}\right)
$$


is the RPA self-energy of the single-particle state $\psi_{k \sigma}(\mathbf{r})$,

$$
g_{\sigma}^{0}\left(\mathbf{r}, \mathbf{r}^{\prime}, i \omega\right)=\frac{1}{\Omega} \sum_{k^{\prime}} \frac{\psi_{k^{\prime} \sigma}(\mathbf{r}) \psi_{k^{\prime} \sigma}^{*}\left(\mathbf{r}^{\prime}\right)}{i \omega-\varepsilon_{k^{\prime} \sigma}}
$$

is the one-particle Green's function for the electrons with spin $\sigma$. The self-energy expression (35b) is the same as those used in usual $(G W)$ self-energy calculations except that the RPA effective interaction is now evaluated in LDA. This is a result of our further approximation that all "local" elements are evaluated in LDA. The average $\left\langle\Sigma_{\sigma}\right\rangle$ is defined as

$$
\left\langle\Sigma_{\sigma}(k ; \mu)\right\rangle=\left[\frac{1}{\Omega} \sum_{k} \delta\left(\mu-\varepsilon_{k \sigma}\right) \Sigma_{\sigma}(k, \mu)\right] N_{\sigma}(0)^{-1},
$$

which is the average self-energy for electrons with spin $\sigma$ on the Fermi surface. The term $\chi^{-1(\text { LDA })}$ [see Eq. (27)] is given by

$$
\begin{aligned}
& \chi_{m \xi_{W}}^{-1(\text { LDA })}(0, \mathbf{q}) \\
& \quad=\left.\frac{1}{\Omega} \int d \mathbf{r} \chi_{m \xi_{W}}^{-1}\left(\mathbf{q}^{\prime} \rightarrow 0\right)\right|_{n=n(\mathbf{r}), m=m(\mathbf{r})} e^{i \mathbf{q} \cdot \mathbf{r}},
\end{aligned}
$$

where $\chi_{m \xi_{W}}^{-1}\left(\mathbf{q}^{\prime} \rightarrow 0\right)$ is the inverse of the $\mathbf{q}^{\prime} \rightarrow 0$ component of the $m-\xi_{W}$ response function for a homogeneous electron gas with densities $n=n(\mathbf{r})$ and $m=m(\mathbf{r})$, evaluated in RPA.

Similarly, $W_{\mathrm{xc}}^{\mathrm{NL}(L D A)}(q)$ is given by

$$
\begin{aligned}
W_{\mathrm{xc}}^{\mathrm{NL}(\mathrm{LDA})}(\mathbf{q}) & \\
=-\mu_{B} \sum_{\sigma} \sigma \frac{1}{\Omega} \int & {\left[\tilde{N} \Sigma_{\sigma}^{\mathrm{RPA}}\left(k_{F \sigma}, \mu\right)\right]_{n=n(r), m=m(r)} } \\
\times & \times d \mathbf{r} \cdot \tilde{\chi}_{m \xi_{W}^{-(L D A)}}^{-1(0, \mathbf{q}),}
\end{aligned}
$$

where the quantity in the square brackets is also evaluated in local-density approximation.

The corresponding approximation for $V_{x c}(0)$ can be derived in a similar way. After some lengthy but similar algebra, we obtain

$$
\begin{aligned}
V_{\mathrm{xc}}(0) \cong & \left\langle\Sigma_{\sigma}^{\mathrm{RPA}}(k, \mu)\right\rangle_{n} \\
& -\left\langle\sum_{\mathrm{q}^{\prime} \in\{Q\}} V_{\mathrm{xc}}\left(\mathbf{q}^{\prime}\right) \rho_{k k}^{\sigma}\left(\mathbf{q}^{\prime}\right)\right\rangle_{n} \\
& -\left\langle\sum_{\mathbf{q}^{\prime} \in\{0, Q\}} \sigma H_{\mathrm{xc}}\left(\mathbf{q}^{\prime}\right) \rho_{k k}^{\sigma}\left(\mathbf{q}^{\prime}\right)\right\rangle_{n},
\end{aligned}
$$

where

$$
\left\langle A_{k, \sigma}\right\rangle_{n}=\left[\frac{1}{\Omega} \sum_{k, \sigma} \delta\left(\mu-\epsilon_{k \sigma}\right) A_{k \sigma}\right] N(0)^{-1}
$$

is the average of $A_{k \sigma}$ over both spin states on the Fermi surface. $N(0)=N_{\uparrow}(0)+N_{\downarrow}(0)$. The terms $V_{\mathrm{xc}}\left(\mathbf{q}^{\prime}\right)$ and $H_{\mathrm{xc}}\left(\mathbf{q}^{\prime}\right)$ in Eq. (37) are given by our approximate expression Eq. (33). Notice that in Green's-function theory, the quasiparticle energy $E_{k \sigma}$ of state $\psi_{k \sigma}(\mathbf{r})$ can be obtained by solving the Dyson's equation, where

$$
\begin{aligned}
E_{k \sigma}= & t(k)+\left\langle V^{\text {ext }}\right\rangle_{k}+\sigma\left\langle H^{\text {ext }}\right\rangle_{k} \\
& +\left\langle V^{H}\right\rangle_{k}+\Sigma_{\sigma}\left(k, E_{k \sigma}\right) .
\end{aligned}
$$

$t(k)$ is the kinetic energy, $\left\langle V^{\mathrm{ext}}\right\rangle_{k}$ and $\left\langle H^{\mathrm{ext}}\right\rangle_{k}$ are the potential energies, and $\left\langle V^{H}\right\rangle_{k}$ is the Hartree self-energy of state $\psi_{k \sigma}$. Also, in density-functional theory, we have for the Kohn-Sham spectrum,

$$
\begin{aligned}
\varepsilon_{k \sigma}= & t(k)+\left\langle V^{\mathrm{ext}}\right\rangle_{k}+\sigma\left\langle H^{\mathrm{ext}}\right\rangle_{k}+\left\langle V^{H}\right\rangle_{k} \\
& +\left\langle V_{\mathrm{xc}}\right\rangle_{k}+\sigma\left\langle H_{\mathrm{xc}}\right\rangle_{k},
\end{aligned}
$$

where $\left\langle V_{\mathrm{xc}}\right\rangle_{k}$ and $\left\langle H_{\mathrm{xc}}\right\rangle_{k}$ are the exchange-correlation potential energies for the state $\psi_{k \sigma}$. Using Eq. (37) for $V_{\mathrm{xc}}(0)$, we found that the chemical potential $\mu$ in our theory is given by an expression identical to the one used in Green's-function theory except for our further localdensity approximation. This is, of course, not merely an accident. It follows from the fact that the chemical potential obtained from the Kohn-Sham theory is in fact the true chemical potential, ${ }^{11,22}$ although $E_{k \sigma} \neq \varepsilon_{k \sigma}$ in general.

Equations (33) - (37) are the main results in this paper. Here we shall make some comments on these results. The main philosphy we kept in deriving our approximate expression for $W_{\mathrm{xc}}(\mathrm{q})$ and $V_{\mathrm{xc}}(0)$ is (1) we assume that LDA provides indeed a good description for the shortrange or "local" exchange-correlation effects around an electron, so we keep this approximation for describing short-range correlations. (2) There is however, a longranged "nonlocal" effect which is absent in LDA, which we have tried also to include in our expression for $W_{\mathrm{xc}}(\mathbf{q})$. Our method of doing this is quite straightforward. We add the nonlocal term $W_{\mathrm{xc}}^{\mathrm{NL}}(\mathrm{q})$ directly to the LDA term and subtract off the corresponding term evaluated in LDA. This procedure is simple, but is probably not the best way to handle the problem. A procedure which interpolate more smoothly between the "local" and "nonlocal" contributions to the exchange-correlation potentials would probably give a better approximation to the exchange-correlation potentials. The problem remains how to do this effectively.

Another approximation which remains to be justified is our "local" approximation for $\chi^{-1}$. This approximation is certainly inaccurate if $\chi$ is dominated by the "nonlocal" terms. In that case, a direct construction of $\chi^{-1}$ keeping only the dominant term in $\chi$ is probably a more reliable approximation.

\section{COMMENTS AND DISCUSSIONS}

In this paper we have analyzed the exchangecorrelation potentials in the Kohn-Sham theory, using an exact expression derived by us. Within RPA, we were able to distinguish between the "local" and "nonlocal" contributions to the exchange-correlation potentials, pin down their physical origin, and construct approximate expressions for the exchange-correlation potential which incorporate approximately the "nonlocal" effects which are missing in LDA. However, several important prob- 
lems remain unanswered. For example, what is technically the "best" way to incorporate both "local" and "nonlocal" contributions in an approximate expression for the exchange-correlation potential? A few comments on this problem were given in the last section. A more fundamental problem concerns the possibility of extension of our theory beyond RPA. In particular, we would like to know whether the qualitative conclusions drawn from our analysis would remain correct in a more "exact" theory. We believe that this is indeed the case, provided that higher-order terms in the Coulomb interaction form a converging perturbation series. The reason is apparent from a diagrammatic viewpoint, since each additional interaction line in a diagram would result in a new integration over all momentum and energy, thus a contribution which is local because the whole Fermi sphere is involved. Thus, the "local" processes in RPA remain "local." Moreover, these "local" contributions would not affect qualitatively the "nonlocal" processes in the exchange-correlation potential as long as the perturbation series converge. In the $q \rightarrow 0$ limit, the effect of higher-order terms in the Coulomb interaction would be to change the noninteracting electrons into quasiparticles, which are described by a Landau-type Fermi-liquid theory. Thus, we believe that our classification of "local" and "nonlocal" processes in the exchange-correlation potentials remains correct in the exact theory, and that the "nonlocal" processes involve only states on the Fermi surface, except that these states on the Fermi surface are “dressed" quasiparticle states, but not states for a noninteracting electron gas. A more difficult question is how our approach can be extended to the situation when electrons are moving in general, nonperiodic potentials where there is no natural separation in length scale, so that the distinction between "local" and "nonlocal" terms is not clear. This is the situation in finite-size systems or systems with a boundary (surface states). So far we have found no simple solution to this problem.

Another difficult problem is how our approach can be extended to the more general situation when magnetic fields are pointing in arbitrary directions in space, since in this case the density-magnetization response function may not be invertible (see Appendix A) and the formal expressions for the exchange-correlation potentials [Eq. (10)] may not be valid. We believe that this is a purely technical matter, since we have also shown (Appendix A) that the density-magnetization response function is invertible at any nonzero finite temperature. Thus, our theory can be formulated at finite temperature, and the problem is how to take the zero-temperature limit properly. In particular, we believe that the distinction of local and nonlocal terms remains valid in the more general situation, since the noninvertibility of the response function at zero temperature is associated with external potentials which have no physical effects on the system only (Appendix A).

In summary, we believe that we have derived a formalism in which the exchange-correlation potentials in density- and spin-density-functional theory can be constructed more systematically. However, work remains to be done to find out what is the best way to construct ap- proximate exchange-correlation potentials in the wide variety of realistic systems.

\section{ACKNOWLEDGMENTS}

This work is supported by the National Science Foundation (NSF) under Grant No. DMR-8521377.

\section{APPENDIX A: INVERTIBILITY OF THE DENSITY-MAGNETIZATION RESPONSE MATRIX $\chi_{\xi \xi^{\prime}}\left(\mathbf{r}, \mathbf{r}^{\prime}\right)$}

In this appendix we consider the generalization of the invertibility theorem of Mearns and $\mathrm{Kohn}^{12}$ to magnetic systems. Using standard linear-response theory, we obtain

$$
\begin{aligned}
\chi_{\xi \xi^{\prime}}\left(\mathbf{r}, \mathbf{r}^{\prime}, \omega\right) & \\
& =\sum_{n=1}^{\infty} \frac{2 E_{n}}{\omega^{2}-E_{n}^{2}}\langle 0|\xi(\mathbf{r})| n\rangle\left\langle n\left|\xi^{\prime}\left(\mathbf{r}^{\prime}\right)\right| 0\right\rangle,
\end{aligned}
$$

where $|0\rangle$ is the ground state of the system and $|n\rangle$ $(n=1, \ldots, \infty)$ are excited states. $E_{n}$ is the excitation energy for state $|n\rangle . \xi, \xi^{\prime}=n, m$.

We shall only consider the regime $\omega<E_{1}$ (i.e., when $\omega$ is less than the first excitation energy of the system ${ }^{12}$ ). In this regime, $\chi_{\xi \xi^{\prime}}\left(\mathbf{r}, \mathbf{r}^{\prime}, \omega\right)$ is a Hermitian and real matrix, and is thus diagonalizable with real eigenvalues. Therefore, to prove that $\chi$ is invertible, it is sufficient to prove that $\chi$ has no zero eigenvalues, or

$$
\sum_{\xi^{\prime}} \int \chi_{\xi \xi^{\prime}}\left(\mathbf{r}, \mathbf{r}^{\prime}, \omega\right) V_{\xi^{\prime}}\left(\mathbf{r}^{\prime}, \omega\right) d \mathbf{r}^{\prime} \neq 0,
$$

for all possible $V_{\xi}(\mathbf{r}, \omega)\left(V_{n}\right.$ is the scalar potential, $V_{m}$ is the magnetic field).

To prove (A2), we consider the quantity

$$
I=\sum_{\xi ; \xi^{\prime}} \int d \mathbf{r} d \mathbf{r}^{\prime} V_{\xi}(\mathbf{r}, \omega) \chi_{\xi \xi^{\prime}}\left(\mathbf{r}, \mathbf{r}^{\prime}, \omega\right) V_{\xi^{\prime}}\left(\mathbf{r}^{\prime}, \omega\right),
$$

which cannot be zero for all possible $V_{\xi}(\mathbf{r}, \omega)$ if (A2) is satisfied.

Using (A1), it is straightforward to obtain

$$
I=\sum_{n=1}^{\infty} \frac{2 E_{n}}{\omega^{2}-E_{n}^{2}}\left|V_{n 0}\right|^{2},
$$

where

$$
V_{n 0}=\sum_{\xi} \int d \mathbf{r}\langle n|\xi(\mathbf{r})| 0\rangle V_{\xi}(\mathbf{r}) .
$$

$I$ is a negative-definite quantity if (i) $V_{n 0}$ is not equal to zero for all excited states $n$ and (ii) $\omega<E_{1}$. Thus, we recover the invertibility theorem of Mearns and Kohn when these conditions are satisfied. ${ }^{12}$

We shall now consider these conditions. Condition (ii) means that the ground state of the system under consideration must be nondegenerate and stable, so that a nonzero $E_{1}>0$ exist, while condition (i) says that the external potentials should be physically "perturbing" the system. Notice that external potentials which do not satisfy condition (i) exist in general. For example, a con- 
stant scalar potential $V=$ const. More generally, external potentials $\mathbf{H}$ and $V$ which satisfy the equation

$$
\int d \mathbf{r}[\mathbf{H}(\mathbf{r}) \cdot \boldsymbol{\sigma}(\mathbf{r})+V(\mathbf{r}) n(\mathbf{r})]|0\rangle=\lambda|0\rangle,
$$

where $\lambda$ is any numerical constant do not satisfy condition (i).

For the special condition considered in this paper $[\mathbf{H}(\mathbf{r})=\hat{\mathbf{z}} \boldsymbol{H}(\mathbf{r})]$, it is easy to see that a nontrivial solution to (A5) does not exist in general, thus proving the invertibility theorem. However, for the general situation $H_{y}, H_{x} \neq 0$, it can also be shown that nontrivial solutions to (A5) exist for a one-particle system with a given ground state $\psi_{\sigma}(\mathbf{r})$ (this result was first obtained by von Barth and $\mathrm{Hedin}^{23}$ ) which leads to the general conclusion that different external potentials can give rise to the same ground-state wave function in the magnetic situation. However, it can also be shown that the densitymagnetization response function is invertible at any finite temperature $T \neq 0$. At finite temperature, (A1) becomes

$$
\begin{aligned}
\chi_{\xi \xi^{\prime}}\left(\mathbf{r}, \mathbf{r}^{\prime}, \omega\right)=\sum_{E_{n}>E_{m}} & \frac{2 E_{n m}}{\omega^{2}-E_{n m}^{2}}\left(e^{-\beta E_{m}}-e^{-\beta E_{n}}\right) \\
& \times\langle m|\xi(\mathbf{r})| n\rangle\left\langle n\left|\xi^{\prime}\left(\mathbf{r}^{\prime}\right)\right| m\right\rangle,
\end{aligned}
$$

where $E_{n m}=E_{n}-E_{m}$, and $\beta=(k T)^{-1}$. This expression is valid for $\omega<\omega_{\min }$, where $\omega_{\min }$ is the minimal energy-level spacing in the system. Repeating the derivation for $T=0$, we find that $\chi_{\xi \xi^{\prime}}\left(\mathbf{r}, \mathbf{r}^{\prime}, 0\right)$ is noninvertible only when $V_{n m}$ is zero for all states $n \neq m$, where

$$
V_{n m}=\sum_{\xi} \int d \mathbf{r}\langle n|\xi(\mathbf{r})| m\rangle V_{\xi}(\mathbf{r})
$$

However, $V_{n m}$ is zero for all states $n \neq m$ only when the operator $V$ commutes with the Hamiltonian of the system, which in the present case is certainly not satisfied, thus proving that $\chi$ is invertible at finite temperature.

Thus, it seems that we are in a very unreasonable situation when $H_{x}, H_{y} \neq 0$, where the density-magnetization response function is invertible at any finite temperature, leading to well-defined exchange-correlation potentials [Eq. (10)]. However, such a procedure may not be valid at zero temperature. This paradoxial situation can be resolved by noticing that the noninvertibility of the response function at zero temperature is always associated with a class of external potentials $W^{\prime}$ which satisfy Eq. (A5). The ground state of the system is not being disturbed upon application of $W^{\prime}$, implying $\delta E_{\mathrm{xc}} / \delta W^{\prime}=0$ at zero temperature. Thus, although individual terms in Eq. (10) may not be well defined in the zero-temperature limit, their product is, leading to a well-defined exchangecorrelation potential in the $T \rightarrow 0$ limit.

In summary, we have shown that the densitymagnetization response function $\chi_{\xi \xi^{\prime}}\left(\mathbf{r}, \mathbf{r}^{\prime}, 0\right)$ is invertible (at zero temperature) under the special situation $\mathbf{H}(\mathbf{r})=\hat{\mathbf{z}} H(\mathbf{r})$, and is also invertible for the general situation at finite temperature. Moreover, the noninvertibility of the response function in the general situation at zero temperature is associated with external potentials which have no physical effects on the system.

\section{APPENDIX B: RANDOM-PHASE APPROXIMATION FOR THE CORRELATION FUNCTIONS}

In RPA, a three-particle correlation function can be decomposed as shown in Fig. 1. Writing it down explicitly, using Feynman's rules, ${ }^{18,24}$ we obtain

$$
\begin{array}{r}
\chi_{\lambda n n ; n}\left(\mathbf{q}^{\prime}, \mathbf{q}-\mathbf{q}^{\prime}, \mathbf{q}^{\prime \prime}\right)=\frac{1}{\beta} \sum_{i \Omega_{n} \mathbf{Q}^{\prime}, \mathbf{Q}^{\prime \prime} \in\{0, Q\}} \mathbf{Q}^{\prime \prime \prime} \in\{Q\} \\
\frac{\epsilon\left(\mathbf{q}^{\prime}, \mathbf{Q}^{\prime}+\mathbf{q}^{\prime}, i \Omega_{n}\right)}{\epsilon\left(\mathbf{q}-\mathbf{q}^{\prime}, \mathbf{Q}^{\prime \prime}-\mathbf{q}^{\prime},-i \Omega_{n}\right)} \\
\times \frac{\chi_{0 n n ; n}\left(\mathbf{Q}^{\prime}+\mathbf{q}^{\prime}, i \Omega_{n}, \mathbf{Q}^{\prime \prime}-\mathbf{q}^{\prime},-i \Omega_{n}, \mathbf{Q}^{\prime \prime \prime}, 0\right)}{\epsilon\left(\mathbf{Q}^{\prime \prime \prime}, \mathbf{q}^{\prime \prime}, 0\right)},
\end{array}
$$

where

$$
\frac{1}{\epsilon\left(\mathbf{q}, \mathbf{q}^{\prime}, \omega\right)}=\delta\left(\mathbf{q}-\mathbf{q}^{\prime}\right)+\phi(\mathbf{q}) \chi_{n n}\left(\mathbf{q}, \mathbf{q}^{\prime}, \omega\right)
$$

is the inverse of the dynamical dielectric function $\epsilon\left(\mathbf{q}, \mathbf{q}^{\prime}, \omega\right) . \quad \chi_{n n}\left(\mathbf{q}, \mathbf{q}^{\prime}, \omega\right)$ is the frequency-dependent density-density response function which is given in RPA by

$$
\begin{aligned}
\chi_{n n}\left(\mathbf{q}, \mathbf{q}^{\prime}, \omega\right) & \\
=\sum_{\mathbf{Q}^{\prime} \in\{0, Q\}} & \chi_{0 n n}\left(\mathbf{q}, \mathbf{Q}^{\prime}+\mathbf{q}^{\prime}, \omega\right) \\
& \times\left[\delta\left(\mathbf{Q}^{\prime}\right)+\phi\left(\mathbf{Q}^{\prime}+\mathbf{q}^{\prime}\right) \chi_{n n}\left(\mathbf{Q}^{\prime}+\mathbf{q}^{\prime}, \mathbf{q}^{\prime}, \omega\right)\right],
\end{aligned}
$$

$\phi(\mathbf{q})=4 \pi / q^{2}$ is the Coulomb potential. $\chi_{0 n n ; n}$ and $\chi_{0 n n}$ are the three-particle and two-particle response functions for a system of noninteracting electrons moving in the KS potential, respectively. Notice that the energy transfer between the two vertices with momentum transfer $\mathbf{q}^{\prime}$ and $\mathbf{q}-\mathbf{q}^{\prime}$ is being summed. This is because these two vertices are evaluated at equal time, which implies a complete uncertainty in energy transfer between the two vertices. The matrix $\chi_{n n}\left(\mathbf{q}, \mathbf{q}^{\prime}\right)^{-1}$ can be expressed in terms of the inverse of the corresponding response functions $\chi_{0 n n}\left(\mathbf{q}, \mathbf{q}^{\prime}\right)$ for the noninteracting system of electrons in a straightforward manner. We obtain 
$\chi_{n n}^{-1}\left(\mathbf{q}, \mathbf{q}^{\prime}\right)=\sum_{\mathbf{Q}^{\prime} \in\{0, Q\}} \chi_{0 n n}^{-1}\left(\mathbf{q}, \mathbf{Q}^{\prime}+\mathbf{q}^{\prime}\right)$

$$
\times\left[\delta\left(\mathbf{Q}^{\prime}\right)-\chi_{0 n n}\left(\mathbf{Q}^{\prime}+\mathbf{q}^{\prime}, \mathbf{q}^{\prime}\right) \phi\left(\mathbf{q}^{\prime}\right)\right]
$$

Combining (B1) and (B4) into Eqs. (17) and (18), Eqs. (19) and (20) can be obtained rather easily.

\section{APPENDIX C: DERIVATION OF EQ. (34) FOR $W_{\mathrm{xc}}^{\mathrm{NL}}(q)$}

To derive Eq. (34) from Eq. (32), first of all, we rewrite Eq. (32) in terms of $\mathbf{r}$ coordinates:

$$
\begin{aligned}
& \left.W_{\mathbf{x c}}^{\mathrm{NL}}(\mathbf{q})\right|_{\mathbf{q} \in\left\{\xi_{W}\right\}} \\
& =\frac{1}{2 \Omega} \int d \mathbf{r}_{1} \int d \mathbf{r}_{2} \frac{1}{\left|\mathbf{r}_{1}-\mathbf{r}_{2}\right|} \int_{0}^{1} d \lambda \frac{1}{\beta} \sum_{i \Omega_{n}} \int d \mathbf{r}_{3} \int d \mathbf{r}_{4}\left(\frac{1}{\epsilon_{\lambda}\left(\mathbf{r}_{1}-\mathbf{r}_{3}, i \Omega_{n}\right)} \frac{1}{\epsilon_{\lambda}\left(\mathbf{r}_{2}-\mathbf{r}_{4}, i \Omega_{n}\right)}\right)_{n=n\left(\mathbf{r}_{1}\right), m=m\left(\mathbf{r}_{1}\right)} \\
& \times\left(\mu_{B} \sum_{\sigma}(\delta-\sigma) \frac{\partial}{\partial \mu_{\sigma}} \chi_{0 \sigma}\left(\mathbf{r}_{3}, \mathbf{r}_{4}, i \Omega_{n}\right)\right] \widetilde{\chi}_{\lambda m \xi_{W}}^{-1(\mathrm{LDA})(0, \mathbf{q})},
\end{aligned}
$$

where we have made use of Eq. (28). Notice that there is an arbitrariness in the choice of local densities $n$ and $m$ in LDA. $n=n\left(\mathbf{r}_{2}\right), n=n\left(\mathbf{r}_{3}\right)$, etc. are equally valid in LDA. The term $\widetilde{\chi}_{\lambda m \xi_{W}}^{-1(\mathrm{LDA})}(0, \mathbf{q})$ is given by Eqs. (27) and (35e). Notice that there is no correlation in $\mathbf{r}$ space between this term and the rest of the integral (nonlocality), in contrast with usual LDA where all terms in Eq. (C1) are evaluated at fixed densities $n=n\left(\mathbf{r}_{1}\right)$ and $m=m$ ( $\left.\mathbf{r}_{1}\right)$, i.e., the correlation is always "local" in LDA. Notice also that the $1 / \epsilon$ terms in Eq. (C1) are inverse dielectric functions of homogeneous electron gas now.

It can be shown by direct construction that within RPA the inverse response function $\chi_{m \xi_{W}}^{-1}\left(\mathbf{q}^{\prime}\right)$ is independent of $\phi\left(\mathbf{q}^{\prime}\right)$ (or $\lambda$ ) for a homogeneous electron gas. Thus the coupling constant integral in Eq. (C1) can be performed directly, resulting in

$$
\begin{aligned}
\left.W_{\mathrm{xc}}^{\mathrm{NL}}(\mathbf{q})\right|_{\mathbf{q} \in\left\{\xi_{W}\right\}}=\frac{1}{2 \Omega} \int d \mathbf{r}_{1} \int d \mathbf{r}_{2} \frac{1}{\beta} \sum_{i \Omega_{n}} & \left.\left.V_{\mathrm{eff}}^{\mathrm{RPA}}\left(\mathbf{r}_{1}-\mathbf{r}_{2}, i \Omega_{n}\right)\right|_{n=n\left(\mathbf{r}_{1}\right), m=m\left(\mathbf{r}_{1}\right)} \mid \mu_{B} \sum_{\sigma}(\delta-\sigma) \frac{\partial}{\partial \mu_{\sigma}} \chi_{0 \sigma}\left(\mathbf{r}_{1}, \mathbf{r}_{2}, i \Omega_{n}\right)\right) \\
& \times \tilde{\chi}_{m \xi_{W}^{-1(\mathrm{LDA})}(0, \mathbf{q} \rightarrow 0),}
\end{aligned}
$$

where $V_{\mathrm{eff}}^{\mathrm{RPA}}(\mathbf{q}, \omega)=\phi(\mathbf{q}) / \epsilon(\mathbf{q}, \omega)$ is the dynamically screened electron-electron effective interaction for a homogeneous electron gas. To proceed further, we observe that the term $(\partial / \partial \mu)\left[\chi_{0 \sigma}\left(\mathbf{r}_{1}, \mathbf{r}_{2}, i \Omega_{n}\right)\right]$ can be rewritten [using Eq. (31)] as

$$
\begin{aligned}
\frac{\partial}{\partial \mu} \chi_{0 \sigma}\left(\mathbf{r}_{1}, \mathbf{r}_{2}, i \Omega_{n}\right) & =\frac{-1}{\Omega^{2}} \sum_{k, k^{\prime}} \delta\left(\mu-\varepsilon_{k \sigma}\right)\left[\frac{\psi_{k \sigma}\left(\mathbf{r}_{1}\right) \psi_{k \sigma}^{*}\left(\mathbf{r}_{2}\right) \psi_{k^{\prime} \sigma}\left(\mathbf{r}_{2}\right) \psi_{k^{\prime} \sigma}^{*}\left(\mathbf{r}_{1}\right)}{\mu-\varepsilon_{k^{\prime} \sigma}-i \Omega_{n}}+\frac{\psi_{k^{\prime} \sigma}\left(\mathbf{r}_{1}\right) \psi_{k^{\prime} \sigma}^{*}\left(\mathbf{r}_{2}\right) \psi_{k \sigma}\left(\mathbf{r}_{2}\right) \psi_{k \sigma}^{*}\left(\mathbf{r}_{1}\right)}{i \Omega_{n}+\mu-\varepsilon_{k^{\prime} \sigma}}\right) \\
& =\frac{-1}{\Omega} \sum_{k} \delta\left(\mu-\varepsilon_{k \sigma}\right)\left[g_{\sigma}^{0}\left(\mathbf{r}_{2}, \mathbf{r}_{1}, \mu-i \Omega_{n}\right) \psi_{k \sigma}\left(\mathbf{r}_{1}\right) \psi_{k \sigma}^{*}\left(\mathbf{r}_{2}\right)+g_{\sigma}^{0}\left(\mathbf{r}_{1}, \mathbf{r}_{2}, \mu+i \Omega_{n}\right) \psi_{k \sigma}\left(\mathbf{r}_{2}\right) \psi_{k \sigma}^{*}\left(\mathbf{r}_{1}\right)\right],
\end{aligned}
$$

where $g_{\sigma}^{0}$ is the one-particle Green's function as defined in Eq. (35c). Substituting Eq. (C3) into (C2), and observing that $\Sigma(k, \mu)$ is real on the Fermi surface, we obtain Eq. (34).

${ }^{1}$ P. Hohenberg and W. Kohn, Phys. Rev. 136, B864 (1964).

${ }^{2}$ W. Kohn and L. J. Sham, Phys. Rev. 140, A1133 (1965).

${ }^{3}$ See, for example, W. E. Pickett, Comments Solid State Phys. 12, 2 (1986).

${ }^{4}$ L. J. Sham and M. Schlüter, Phys. Rev. Lett. 51, 1888 (1983).

5J. P. Perdew and M. Levy, Phys. Rev. Lett. 51, 1884 (1983).

${ }^{6}$ See, for example, J. F. Janak and A. R. Williams, Calculated Electronic Properties of Metals (Pergamon, New York, 1978).

${ }^{7}$ C. S. Wang, B. M. Klein, and H. Krakauer, Phys. Rev. Lett. 54, 1852 (1985)

${ }^{8}$ D. C. Langreth and J. P. Perdew, Phys. Rev. B 15, 2884 (1977).

${ }^{9}$ J. Harris and R. O. Jones, J. Phys. F 4, 1170 (1974).

${ }^{10}$ O. Gunnarsson and B. I. Lundquist, Phys. Rev. B 13, 4274 (1976).

${ }^{11}$ For a review, see Theory of Inhomogeneous Electron Gas, edited by S. Lundqvist and N. H. March (Plenum, New York, 1983).

${ }^{12}$ D. Mearn and W. Kohn, Phys. Rev. A 35, 4796 (1987).

${ }^{13}$ See, for example, E. M. Lifshitz and L. P. Pitaevskii, Statisti- cal Physics, Part II (Pergamon, New York, 1980).

${ }^{14}$ L. J. Sham, Phys. Rev. B 32, 3876 (1985).

${ }^{15}$ See, for example, J. R. Smith, Phys. Rev. 181, 522 (1969).

${ }^{16}$ D. C. Langreth and M. J. Mehl, Phys. Rev. B 28, 1098 (1983).

${ }^{17}$ See, for example, Density Functional Methods in Physics (Plenum, New York, 1985).

${ }^{18}$ See, for example, P. Nozières, Theory of Interacting Fermi Systems, edited by R. M. Dreizler and J. da Providencia (Benjamin, New York, 1964).

${ }^{19}$ T. K. Ng and M. Huang (unpublished).

${ }^{20}$ M. Lannoo, M. Schlüter, and L. J. Sham, Phys. Rev. B 32, 3890 (1985).

${ }^{21}$ G. Strinati, H. J. Mattausch, and W. Hanke, Phys. Rev. B 25, 2867 (1982).

${ }^{22}$ T. C. Koopmans, Physica 1, 104 (1933).

${ }^{23}$ U. von Barth and L. Hedin, J. Phys. C 5, 1629 (1972).

${ }^{24}$ See, for example, G. D. Mahan, Many-Particle Physics (Plenum, New York, 1981). 